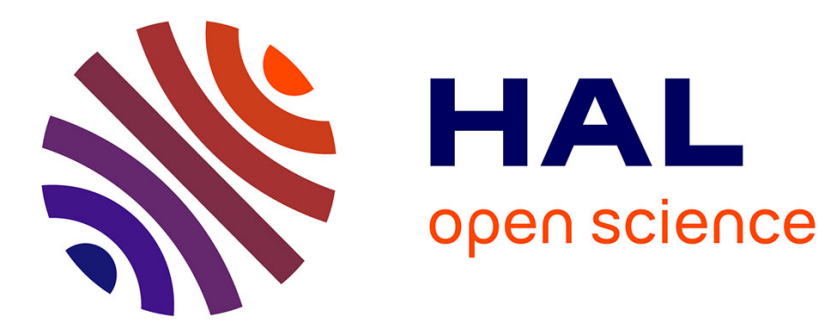

\title{
Channel-Aware Energy Optimization of OFDM Receivers Using Dynamic Precision Scaling in FPGAs
}

Fernando Cladera, Matthieu Gautier, Olivier Sentieys

\section{To cite this version:}

Fernando Cladera, Matthieu Gautier, Olivier Sentieys. Channel-Aware Energy Optimization of OFDM Receivers Using Dynamic Precision Scaling in FPGAs. European Signal Processing Conference (EUSIPCO 2015), Aug 2015, Nice, France. hal-01175917

\section{HAL Id: hal-01175917 https://hal.science/hal-01175917}

Submitted on 15 Jul 2015

HAL is a multi-disciplinary open access archive for the deposit and dissemination of scientific research documents, whether they are published or not. The documents may come from teaching and research institutions in France or abroad, or from public or private research centers.
L'archive ouverte pluridisciplinaire HAL, est destinée au dépôt et à la diffusion de documents scientifiques de niveau recherche, publiés ou non, émanant des établissements d'enseignement et de recherche français ou étrangers, des laboratoires publics ou privés. 


\title{
CHANNEL-AWARE ENERGY OPTIMIZATION OF OFDM RECEIVERS USING DYNAMIC PRECISION SCALING IN FPGAS
}

\author{
Fernando Cladera, Matthieu Gautier and Olivier Sentieys \\ University of Rennes 1, IRISA, INRIA, France
}

\begin{abstract}
To reduce the energy consumption of Orthogonal FrequencyDivision Multiplexing (OFDM) systems, a new variable word-length method is presented in this paper. A simulation based approach is used: the optimized fixed-point implementation of an OFDM receiver is found for different simulated channel conditions, depending on the Signal-to-Noise Ratio (SNR) and the channel type. During the execution, the receiver estimates the channel conditions and chooses the optimum word-length to decode the received information. A realistic energy consumption of the receiver is estimated with a library that contains the energy consumption of Field-Programmable Gate Array (FPGA) basic operators depending on the bit-width, obtained from experimental data. Up to $57 \%$ of the dynamic energy can be saved using this method.
\end{abstract}

Index Terms - Fixed-point arithmetic, OFDM receiver, energy reduction, variable wordlength

\section{INTRODUCTION}

Wireless devices are ubiquitous nowadays. They are used not only for audio communications, but also new services (such as video streaming) are proposed. These services require high throughput, which leads to an inevitable rise of power consumption. Yet, wireless devices are often battery powered, hence low power consumption is required. This study focusses on wireless systems based on OFDM, which is a standard for a wide range of modern wireless devices (e.g. LTE, 802.11a/g/n, DVB, DAB). The main advantage of OFDM systems is the high immunity to interferences, especially in the case of multipath channels [1].

Fixed-point arithmetic is favored to implement algorithms in embedded systems. Fixed-point operators are faster and use less area and energy. Memory and bus sizes are smaller too [2]. The fixedpoint implementation is chosen during the development of the architecture, using analytical or simulation approaches. For both cases hypotheses are made about the system's working conditions, which influence the resulting word-length of the implementation. Therefore, multiple conditions may imply different fixed-point implementations.

In a static fixed-point wireless receiver, data and operator bitwidths are chosen to deal with the worst condition. However, the system is oversized when better working conditions are present. In [3], Dynamic Precision Scaling (DPS) was introduced: multiple channel conditions are analyzed during the design phase and then, the best fixed-point implementations is chosen during the execution. [3] shows that an energy saving of $25 \%$ to $50 \%$ can be achieved in a WCDMA receiver.

In [4], a new variable adaptive word-length OFDM receiver was presented, reducing the energy consumption by $18 \%$ to $30 \%$. No previous knowledge about the channel condition is needed.
Nonetheless, this technique leads to an unavoidable modification of the OFDM frame by the insertion of a search symbol. Thus, existing standards have to be modified. This technique was improved in [5], by using a Viterbi decoder. The Hamming distance for a maximum word-length receiver is compared with many reduced-precision implementations, to determine the minimum word-length which does not degrade the link. Yet, this method requires many iterative operations during the execution, consuming part of the energy saved. In [6], a dynamic OFDM receiver for software defined radios is presented. Intensive simulations are used to choose the right number of bits for the Fast Fourier Transform (FFT) depending on the SNR, saving $50 \%$ of the energy. The method used to optimize the receiver is similar to the one presented in this paper, but [6] does not specify how to switch between fixed-point implementations and only Additive Gaussian White Noise (AGWN) channels are analyzed. In [7], an analytical method to optimize the FFT block word-length is presented and applied to a DPS system. This method decreases significantly the time needed to find the fixed-point implementations for each channel condition. However, only AGWN channels are targeted, and the energy consumption when channel type varies is not analyzed. Important energy reductions can be achieved if the channel type is considered in the analysis of the word-length.

The contributions of this paper are the following: apply the DPS technique in an OFDM receiver, where a low complexity selector is used to choose the processing word-length at run time (without modifying the standard OFDM frame). Energy savings will be estimated with a library that contains the energy consumption of basic operators (such as adders and multipliers) depending on the bit-width, obtained from experimental data with FPGAs.

This paper is organized as follows: in Section 2, the OFDM receiver and the energy saving strategy will be presented. The energy consumption estimation of the receiver is also tackled. In Section 3 , the fixed-point OFDM model and the fixed-point implementation selector are presented. In Section 4 the DPS architecture is simulated and the results are commented. Finally, Section 5 draws conclusions.

\section{SYSTEM MODEL}

\subsection{Floating-Point Model of the OFDM Receiver}

A frame-based OFDM receiver was proposed for this study with the following parameters: 16-QAM modulation, 512-point FFT $\left(N_{F F T}\right), 300$ used subcarriers and 128-point Cyclic Prefix $\left(N_{c y p}\right)^{1}$. At least one training symbol and ten OFDM symbols were used in an OFDM frame. In this study, the synchronization was supposed perfect and the equalizer coefficients were supposed known. Two channels were analyzed: an one-path AGWN and a Frequency Selective Fading (FSF) channel with AGWN. For both channels, the

\footnotetext{
${ }^{1}$ These parameters have been set in order to emulate a $5 \mathrm{MHz}$ long Cyclic Prefix LTE receiver.
} 


\begin{tabular}{c|cccccccccccc}
\multirow{2}{*}{ Operation } & \multicolumn{1}{c}{ word-length $w l$} \\
& 4 & 5 & 6 & 7 & 8 & 9 & 10 & 11 & 12 & 13 & 14 & 15 \\
\hline ADD & 1.08 & 1.37 & 1.66 & 1.95 & 2.25 & 2.54 & 2.83 & 3.12 & 3.41 & 3.70 & 3.99 & 4.28 \\
\hline LUT MULT & 5.66 & 9.88 & 15.58 & 22.90 & 31.96 & 42.89 & 55.80 & 70.79 & 87.98 & 107.44 & 129.29 & 153.60
\end{tabular}

Table 1. Energy consumed for arithmetic operations (adders and LUT multipliers) in a Virtex-5 FPGA (in $[p J]$ ).

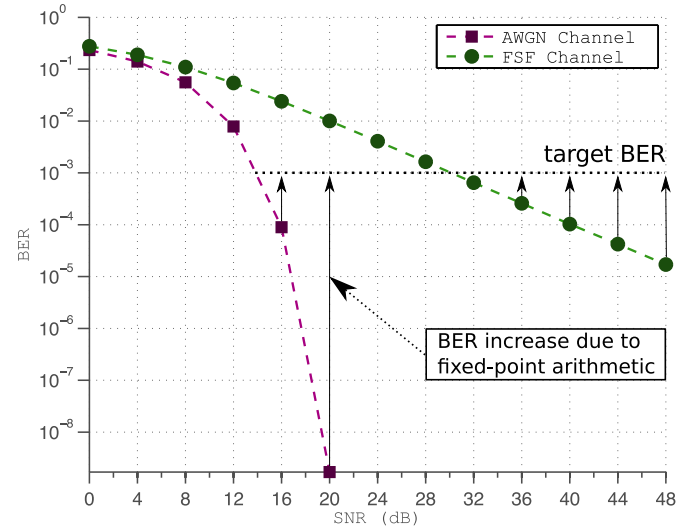

Fig. 1. SNR-BER plot for the floating-point receiver and energy saving strategy.

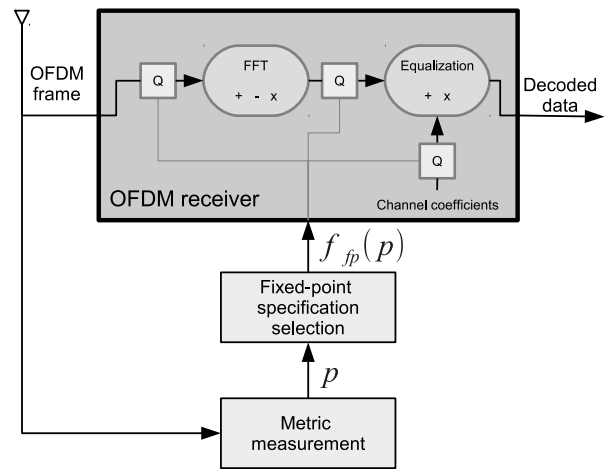

Fig. 2. Dynamic precision scaling algorithm and fixed-point OFDM receiver.

noise level goes from $0 \mathrm{~dB}$ to $48 \mathrm{~dB}$. The FSF channel has 9 paths with an exponential delay profile $(16 \mathrm{~dB}$ between the first and the last path). The total length is $\approx 0.3 N_{\text {cyp }}$. This channel is similar to the Extended Vehicular A Model from [8]. In order to reduce the number of simulations, only one FSF channel has been considered in this study.

\subsection{Energy Saving Strategy}

Fig. 1 shows the curve that links Bit Error Rate (BER) with Signalto-Noise Ratio (SNR), for the floating-point model ${ }^{2}$. Both curves reach very small BER values when the SNR is high.

However, a specific application requires just a minimum BER to guarantee its correct operation. Thus, the receiver presented in this work will target a fixed BER of $10^{-3}$. The use of reduced-precision fixed-point arithmetic increases the BER and saves energy. Fig. 1

${ }^{2}$ All the simulations in this work were executed using MATLAB. also shows the potential gain of this method: worst conditions need wider word-lengths to be as close as possible to the floating-point model, whereas the word-length can be reduced in better channel conditions.

Some points cannot respect the objective BER (low SNR values). Those points will not be optimized and the widest word-length will be used. Usually an error correcting block (e.g. Viterbi and convolutional coding) is included at the end of the receiver. Indeed [9] shows that by using a convolutional coding, the BER decreases considerably, making our system suitable for applications where the quality of the link should be higher.

Fig. 2 synthesizes the algorithm of the receiver: a metric $p$ is calculated from the signal input and a fixed-point implementation is chosen depending on the metric, defined by the function $f_{f p}(p)$, to target the objective BER.

\subsection{Energy Consumption Estimation}

In order to estimate the energy savings of a variable word-length receiver, a cost function $C(w l)$ is used. This cost function is obtained from experiments, measuring the energy spent to perform one operation (multiplication or addition). Even when other elements (such as memories and buses) should be considered to obtain a precise cost function, this rough energy consumption estimator of arithmetic operators can be used to evaluate the savings of our optimized receiver.

FPGAs are configurable architectures used for implementing signal processing algorithms. They allow to modify the interconnection between specialized blocks, Look-Up Tables (LUTs) and registers to achieve more complex structures. Indeed, the wordlength of the operators can be also chosen. To estimate the cost function $C(w l)$, special architectures with a high number of operators were synthesized in a Virtex-5 XC5VLX50T FPGA. Those operators were fed with data from a Pseudo-Random Binary Sequence (PRBS) to ensure maximum activity. The energy is measured using the technique developed in [10].

Table 1 shows the cost function for adders and multipliers, where the dynamic energy consumption of each operator varies with the word-length.

\section{DPS FOR OFDM RECEIVERS}

\subsection{Fixed-Point Model}

Because the synchronization and channel estimation blocks were not considered in this study, our receiver has only two important blocks: the FFT and the equalizer. In order to emulate the fixed-point architecture, three quantizers were introduced in the floating-point receiver limiting the dynamic (saturation) and reducing the precision, as shown by Fig. 2: (1) Receiver input, (2) FFT output, and (3) Channel coefficients.

The optimized word-length in each quantizer is found for each channel condition during the design of the receiver. The word-length is composed of an integer and a fractional part. 


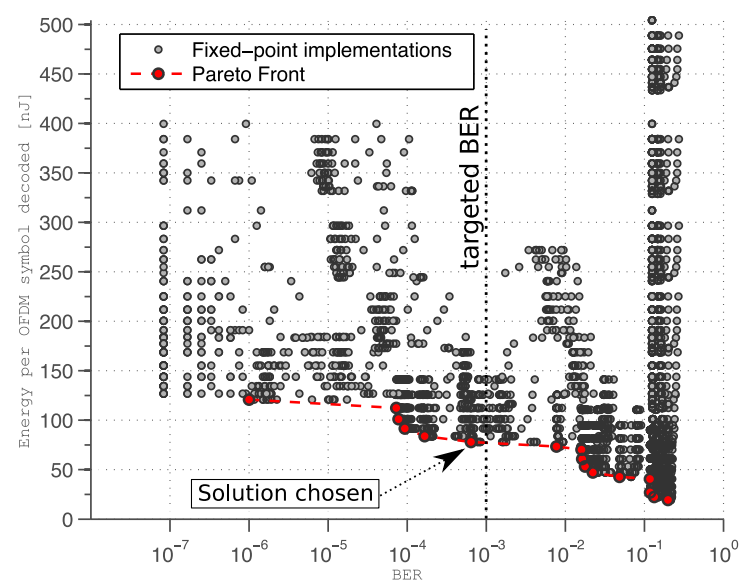

Fig. 3. Fixed-point implementation selection for an AGWN channel and a SNR of $24 \mathrm{~dB}$, for a Virtex-5 FPGA.

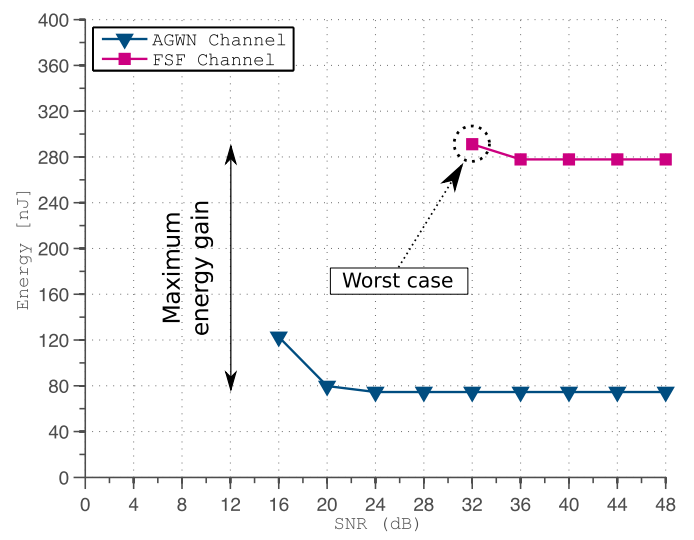

Fig. 4. Energy consumption per OFDM symbol for an optimized fixed-point receiver. The energy depends on the SNR and the channel type.

The dynamic range (integer part) can be easily obtained using a simulation approach: for each channel type and SNR, multiple simulations using the floating point receiver are carried out. The data at each point where a quantizer will be placed is stored. Then, the number of bits that allows to represent the integer part of least 99.9\% of the values is chosen for each quantizer.

However, obtaining the fractional part implies a much more complicated process: for every channel type and SNR, multiple simulations were executed using different fractional parts for each quantizer. Each precision implies a given BER, and the precision chosen is a tradeoff between the quality of the link and the energy spent during the reception. Fig. 3 shows an example of this process, where the energy consumption is related with the decoding quality, for different fractional sizes at each quantizer. Each point represents a different fixed-point implementation of the receiver, with an associated BER and energy consumption. The total energy consumed for a specific solution can be estimated using the data from Section 2.3, knowing the number of operators. The FFT needs $13 \times 10^{3}$ real additions and $9 \times 10^{3}$ real multiplications for this receiver, whereas the equalizer needs 600 real additions and 1200 real multiplications. The less consuming solution that respects a BER of $10^{-3}$ is chosen.

Fig. 4 shows the energy consumed for each optimized fixedpoint solution, depending on the channel conditions. Some prelimi- nary conclusions can be drawn: in the worst case, the energy needed to process an OFDM symbol is $291.05 n \mathrm{~J}$, while the best case needs only $74.56 n J$. Up to $74 \%$ of the energy can be saved using an adaptive receiver. Also, the fixed-point selector has to consider both the SNR and the channel type to calculate the performance metric $p$, used to select one of the five fixed-point implementations.

\subsection{Fixed-Point Implementation Selector}

Section 3.1 shows that the fixed-point implementations depends not only on the SNR but also on channel type. Thus, both parameters have to be estimated to select the appropriate fixed-point implementation. Estimators will be updated with each new symbol received.

\subsubsection{SNR estimation}

The estimator from [11] was used due to its simplicity. The SNR estimation is calculated as:

$$
S N R=\frac{\sigma_{s}^{2}}{\sigma_{n}^{2}}=\left(\frac{y_{a c c}^{\max }}{y_{a c c}^{\min }}\right)^{2}-1,
$$

where $\sigma_{s}^{2}$ is the power of the desired signal and $\sigma_{n}^{2}$ is the power of the noise. $y_{a c c}^{\max }$ and $y_{a c c}^{\min }$ correspond to a sum of $N_{a c c}$ points after subtracting the signal delayed $N_{F F T}$ to the current symbol (minimum and maximum values respectively). In this work, the sum is made from the end to the beginning of the Cyclic Prefix (CP), to avoid the effects of multipath channels. For instance, to calculate $y_{a c c}^{\max }$ the following operation is made:

$$
\begin{aligned}
y_{a c c}^{\max } & =\sum_{N_{c y p}-N_{a c c}}^{N_{c y p}}\left|x_{s u b t}^{\max }\right| \\
& =\sum_{N_{c y p}-N_{a c c}}^{N_{c y p}} \sqrt{\Re\left(x_{s u b t}^{\max }\right)^{2}+\Im\left(x_{\text {subt }}^{\max }\right)^{2}},
\end{aligned}
$$

where $x_{\text {subt }}^{\max }$ is the complex signal after the subtraction. The equivalent equation can be written for $y_{a c c}^{\min }$ using $x_{s u b t}^{\min }$.

Given that the calculation of the absolute value requires a square root, which is complex operation in embedded devices, the following modification is proposed:

$$
\begin{aligned}
& y_{a c c}^{\max *}=\sum_{N_{c y p}-N_{a c c}}^{N_{c y p}}\left|x_{s u b t}^{\max }\right|^{2} \\
& =\sum_{N_{c y p}-N_{a c c}}^{N_{c y p}} \Re\left(x_{\text {subt }}^{\max }\right)^{2}+\Im\left(x_{\text {subt }}^{\max }\right)^{2} \\
& y_{a c c}^{\min *}=\sum_{N_{c y p}-N_{a c c}}^{N_{c y p}}\left|x_{s u b t}^{\min }\right|^{2} \\
& =\sum_{N_{c y p}-N_{a c c}}^{N_{c y p}} \Re\left(x_{\text {subt }}^{\min }\right)^{2}+\Im\left(x_{\text {subt }}^{\text {min }}\right)^{2} \\
& S N R^{*}=\frac{y_{a c c}^{\max *}}{y_{a c c}^{\min *}}-1
\end{aligned}
$$


The number of points $N_{a c c}$ is chosen to target a specific detection error. In this study, we use all the available points of the $\mathrm{CP}$ which have not been afected by the multipath channel. Therefore, $N_{a c c}=90$ points were used to estimate the SNR.

The number of operations needed is 721 real additions, 360 real multiplications and one division. Using a fixed word-length (12-bit), the energy consumed by the SNR estimator is $35.01 \mathrm{~nJ}$ per OFDM symbol $^{3}$.

\subsubsection{Channel Type Estimation}

The variance of the equalizer coefficients was used as an indicator of the channel type. Indeed, in AGWN channels, equalizer coefficients are flat, whereas in FSF channels the coefficients show peaks and depressions. The estimator is given by:

$$
\hat{\theta}=\frac{1}{N} \sum_{i=1}^{N}\left(e q_{i}-\overline{e q_{i}}\right)^{2},
$$

where $e q_{i}$ are the absolute values of the coefficients of the equalizer and $\overline{e q_{i}}$ is the mean of $e q_{i} . \frac{1}{N}$ is a constant that can be eliminated, changing the detection threshold of the estimator.

In order to avoid the calculus of the absolute value (because of the square root), the following modification is proposed:

$$
\hat{\theta}^{*}=\sum_{i=1}^{N}\left|e q_{i}^{2}-\overline{e q_{i}^{2}}\right| .
$$

In addition, the equalizer coefficients are undersampled in order to reduce the number of calculations:

$$
\hat{\theta}^{* *}=\sum_{i=n K}\left|e q_{i}^{2}-\overline{e q_{i}^{2}}\right| \quad n=1,2, \ldots
$$

The undersampling $K$ is chosen to have a specific detection error. In this paper, 75 points are needed to distinguish between different channel types ${ }^{4}$. Thus, 300 real additions, 150 real multiplications and 1 real division are needed for this estimator. Using a fixed wordlength (12-bit), the energy needed is $15.10 \mathrm{~nJ}$ per OFDM symbol.

\section{PERFORMANCE RESULTS}

\subsection{Selector Performance}

One of the keys for the correct behaviour of the dynamic receiver is an accurate fixed-point implementation selector. After simulating both estimators presented in Sec. 3.2, some results are presented in this section.

The SNR estimator presents some wrong detections during the execution. Fig. 5 shows the ratio of missed SNR estimations, depending on the channel type and SNR. The number of points for SNR estimation $N_{a c c}=90$ was chosen to have a ratio of $10^{-3}$ approximately. Experimentally, the average ratio fluctuates between $10^{-2.8}$ for the AGWN channel and $10^{-2.5}$ for the FSF channel.

\footnotetext{
${ }^{3}$ For the sake of simplicity, one division is considered equivalent to ten multiplications.

${ }^{4}$ In our receiver, the equalizer coefficients are presumed known. Nonetheless, the channel type estimator was calibrated using a Normalized Linear Mean Squares (NLMS) equalizer, implemented in a floating point receiver.
}

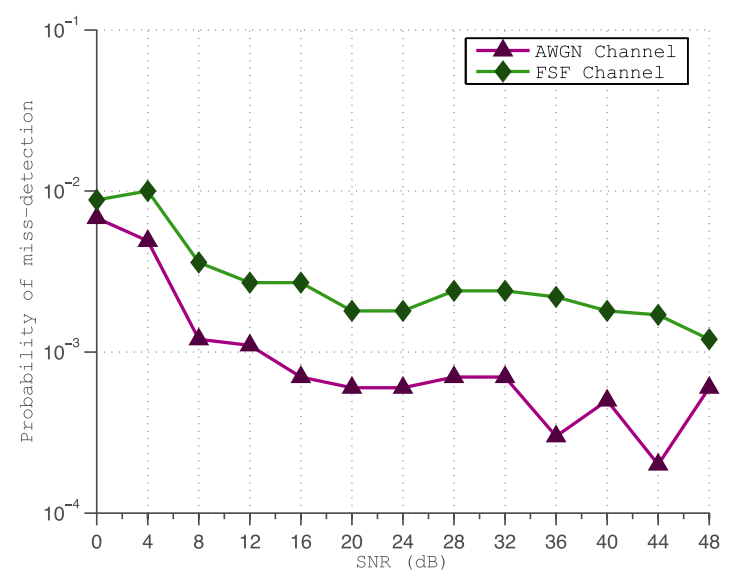

Fig. 5. Probability of miss detection versus SNR of the SNR estimator $\left(N_{a c c}=90\right)$.

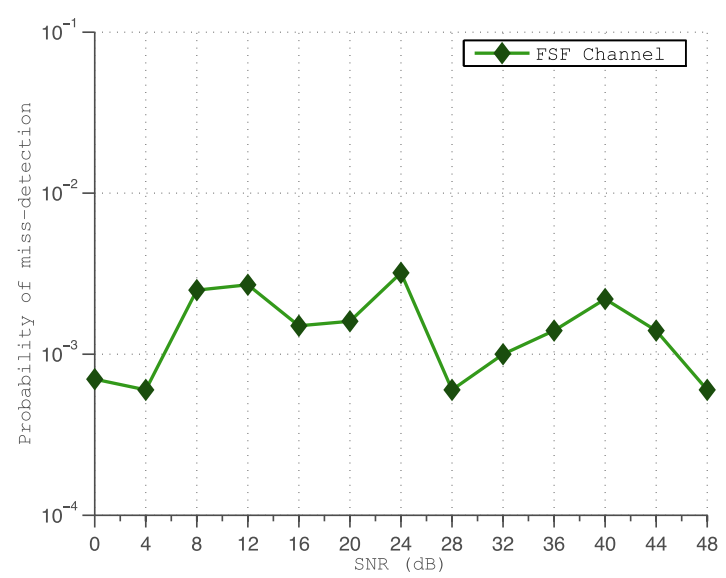

Fig. 6. Probability of miss detection versus SNR of the channel type estimator with 75 points.

The channel type estimator is very accurate with 75 points. Fig. 6 shows the ratio of missed channel type estimations, for the FSF channel. Indeed, due to the fact that equalizer coefficients are known, this estimator is very accurate for AGWN channels. The average probability of miss-detection using 75 points is $10^{-2.8}$ approximately for the FSF channel.

Even if the quality of the SNR estimator seems low for the target $\mathrm{BER}$, this does not mean that the receiver is not working correctly. Indeed, some erroneous SNR detections do not imply a degradation of the link because similar bit-widths may be used.

\subsection{DPS Performance}

Our fixed-point OFDM receiver is combined with the selector, and 1000 simulations have been carried out for each channel condition in order to analyze its behavior. The BER-SNR curve is presented in Fig. 7. Dotted lines correspond to a static receiver using the "worst case" approach, whereas full lines correspond to the dynamic receiver using adaptive precision. It can be observed that for BER $>$ $10^{-3}$ both curves are overlapped. But, when BER $<10^{-3}$ our adaptive system tries to follow the reference value while the static receiver decreases continuously its BER. For the AGWN channel, a superlative quality is reached for high SNR. This is due to the discrete 


\begin{tabular}{cc|ccc|cc}
\multicolumn{2}{c|}{ Fixed-point implementation } & 1 & 2 & 3 & 4 & 5 \\
\hline \multicolumn{2}{c}{ Channel type } & \multicolumn{3}{c|}{ AGWN } & \multicolumn{2}{c}{ FSF } \\
\hline \multicolumn{2}{c|}{ SNR range } & $0-16 \mathrm{~dB}$ & $20 \mathrm{~dB}$ & $24-48 \mathrm{~dB}$ & $0-32 \mathrm{~dB}$ & $36-48 \mathrm{~dB}$ \\
\hline \multirow{2}{*}{ Energy consumption } & {$[n J]$} & 172.81 & 129.90 & 124.66 & 341.16 & 327.86 \\
\cline { 2 - 7 } & {$[\%]$} & $59.4 \%$ & $44.6 \%$ & $42.8 \%$ & $117.2 \%$ & $112.6 \%$
\end{tabular}

Table 2. Energy consumption of the DPS solution compared to a "worst-case" receiver.

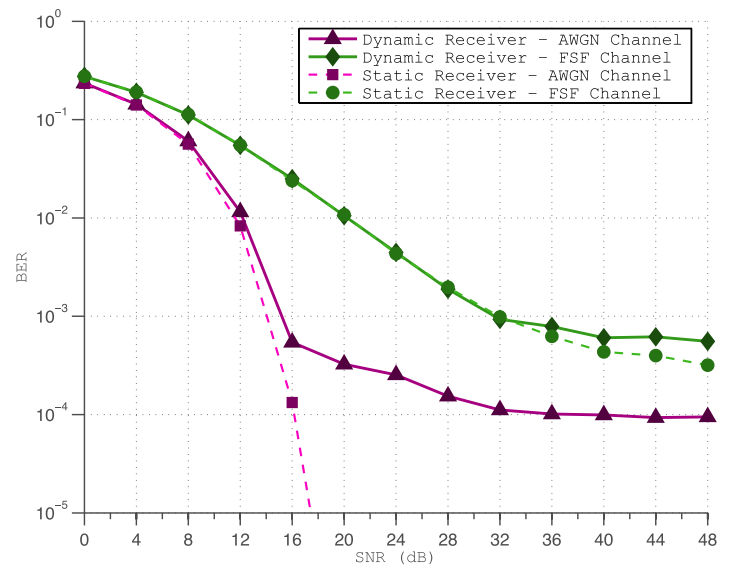

Fig. 7. BER-SNR curve, proving the correct operation of the dynamic receiver.

nature of fixed-point data: the implementation which can achieve the objective BER has this excessive quality.

Tab. 2 shows the energy consumption of the dynamic receiver, with its implementation selector. Comparing the energy consumption of this solution with the "worst case" approach, some conclusions are obtained. The maximum energy saving is approximately $57 \%$. The upper bound defined in Section 3.1 is reduced due to the consumption of the selector. However, when working in FSF channels, the consumption of our receiver is slightly higher: $117 \%$ for low SNR values and $113 \%$ for high SNR values. These results may be improved if the selector uses less energy. For example, calculating the SNR using fewer symbols of the frame.

\section{CONCLUSION}

In this paper, a low power OFDM receiver is developed using DPS. An OFDM receiver is presented, and a procedure to find the appropriate word-length is shown (based on intensive simulations). Results show that the word-length depends not only in the noise level, but also on the channel type. Thus, a low-power selector to switch between the different fixed-point implementations was developed. The energy consumption of the receiver is calculated using a library obtained from our experimental data, knowing the number of operators and the word-length. Compared to the state of the art [4] [5] [6] [7], the OFDM frame is not modified in this method and the introduction of a search symbol is not needed to estimate the channel conditions. Our solution can save up to $57 \%$ of the energy, targeting an objective BER of $10^{-3}$.

\section{REFERENCES}

[1] T.D. Chiueh and P.Y. Tsai, OFDM Baseband Receiver Design for Wireless Communications, John Wiley \& Sons, 2007.

[2] D. Menard, R. Rocher, and O. Sentieys, "Analytical FixedPoint Accuracy Evaluation in Linear Time-Invariant Systems," IEEE Trans. Circuits Syst. I, vol. 55, no. 10, pp. 3197-3208, Nov. 2008.

[3] H. N. Nguyen, D. Menard, and O. Sentieys, "Dynamic precision scaling for low power WCDMA receiver," in Proc. Int. Symp. Circuits Syst. (ISCAS'09), pp. 205-208, Taipei, Taïwan, China, May 2009.

[4] S. Yoshizawa and Y. Miyanaga, "Use of a Variable Wordlength Technique in an OFDM Receiver to Reduce Energy Dissipation," IEEE Trans. Circuits Syst. I, vol. 55, no. 9, pp. 28482859, Oct 2008.

[5] J. Kim, S. Yoshizawa, and Y. Miyanaga, "Dynamic Wordlength Calibration to Reduce Power Dissipation in Wireless OFDM Systems," in IEEE Proc. Asia Pacific Conf. Circuits Syst. (APCCAS'10), pp. 628-631, Kuala Lumpur, Malaysia, 2010.

[6] D. Novo Bruña, Exploiting Adaptive Precision in Software Defined Radios, Ph.D. thesis, Katholieke Universiteit Leuven, Dec. 2007.

[7] S. Lee and A. Gerstlauer, "Fine Grain Word Length Optimization for Dynamic Precision Scaling in DSP Systems," in Proc. Int. Conf. Very Large Scale Integr. (VLSI-SoC'13), Istanbul, Turkey, Oct. 2013.

[8] ETSI, "LTE - Evolved Universal Terrestrial Radio Access (EUTRA) - User Equipment (UE) radio transmission and reception," Technical Specification 3GPP TS 36.101 v. 11.6.0-11, ETSI, 2013.

[9] WAC Fernando, RMAP Rajatheva, and KM Ahmed, "Performance of Coded OFDM with Higher Modulation Schemes," in IEEE Proc. Int. Conf. Comm. Tech. (ICCT'98), vol. 2, pp. 1-5, Beijing, China, Oct. 1998.

[10] R. Bonamy, D. Chillet, S. Bilavarn, and O. Sentieys, "Power Consumption Model for Partial and Dynamic Reconfiguration," in Proc. Int. Conf. Reconf. Comp. and FPGAs (ReConFig'12), pp. 1-8, Cancun, Mexico, Dec. 2012.

[11] L. Wilhelmsson, I. Diaz, T. Olsson, and V. wall, "Analysis of a Novel Low Complex SNR Estimation Technique for OFDM Systems," in IEEE Proc. Int. Conf. Wireless Comm. Netw. (WCNC'11), pp. 1646-1651, Cancun, Mexico, Mar. 2011. 